Diabetologia (1994) 37: 863-870

\title{
The role of islet secretory function in the development of diabetes in the GK Wistar rat
}

\author{
S.J.Hughes ${ }^{1}$, K. Suzuki ${ }^{2}$, Y. Goto ${ }^{2}$ \\ ${ }^{1}$ Division of Basic Medical Science, St. Mary's Hospital Medical School, Imperial College, London, UK \\ ${ }^{2}$ Department of Medicine, Tohoku Medical School, Sendai, Japan
}

Summary Insulin secretion and glucose metabolism were compared in islets isolated from GK Wistar rats (a non-obese, spontaneous model of non-insulin-dependent diabetes mellitus) and control Wistars aged 8 and 14 weeks. By 8 weeks of age, GK Wistar rats were clearly diabetic as indicated by non-fasting plasma glucose concentrations and impaired glucose tolerance. Islet insulin content was not significantly different to controls at either age. In islets from 14-week-old GK Wistar rats glucose-stimulated insulin release (6$16 \mathrm{mmol} / \mathrm{l}$ glucose) was significantly reduced to 25 $50 \%$ of controls in static incubations $(p<0.001)$. In perifusion, glucose-stimulated insulin release was reduced by $90 \%$ for first phase $(p<0.01)$ and by $75 \%$ for second phase $(p<0.05)$. The responses to arginine and $2 \alpha$ Ketoisocaproate in islets were similar to those in controls. In contrast, islets isolated from 8-week-old GK Wistar rats exhibited no significant reduction in glucose-stimulated insulin secretion in static incubations. In perifusion, although both first and second phases of glucose-stimulated insulin release were slightly reduced, these were not significantly different to controls. Islets from 8-week-old GK Wistar rats failed however to respond to stimulation by glyceraldehyde. Raising the medium glucose concentration to $16 \mathrm{mmol} / \mathrm{l}$ significantly increased rates of glucose utilisation $\left(\left[{ }^{3} \mathrm{H}\right] \mathrm{H}_{2} \mathrm{O}\right.$ production from $5-\left[{ }^{3} \mathrm{H}\right]$ glucose $)$ and oxidation $\left(\left[{ }^{14} \mathrm{C}\right] \mathrm{CO}_{2}\right.$ production from $\mathrm{U}-\left[{ }^{14} \mathrm{C}\right]$ glucose) in islets isolated from 8-week-old control and GK Wistar rats, respectively. The rates of oxidation were not significantly different at stimulatory glucose concentrations whereas the rates of utilisation were significantly higher in islets from the diabetic animals $(p<0.05)$. Production of $\left[{ }^{3} \mathrm{H}\right] \mathrm{H}_{2} \mathrm{O}$ from $2-\left[{ }^{3} \mathrm{H}\right]$ glycerol metabolism was increased $(p<0.05)$ at $2 \mathrm{mmol} / \mathrm{l}$ glucose but was not significantly different to controls at $16 \mathrm{mmol} / 1$ glucose in islets from 8-week-old GK Wistar rats. This data would suggest that abnormalities in islet function are present in 8-week-old diabetic animals although these do not seriously impair glucosestimulated insulin release from isolated islets. This in turn would indicate that a defect in the glucose signalling pathway in beta cells is not a primary cause of the diabetes of GK Wistar rats and that deterioration of the secretory response is the consequence of some factor associated with the diabetic condition. [Diabetologia (1994) 37: 863-870]

Key words Non-insulin-dependent diabetes mellitus, pancreatic islet, insulin secretion, glùcose metabolism.
Received: 26 November 1993

and in revised form: 8 April 1994

Corresponding author: Dr. S. J.Hughes, Department of Physiology and Biophysics, St. Mary's Hospital, Medical School, Norfolk Place, London W2 1PG, UK

Abbreviations: KIC, $2 \alpha$ Ketoisocaproate; BSA, bovine serum albumin, GLU'T, glucose transporter.
Type 2 or non-insulin-dependent diabetes mellitus (NIDDM) is characterised by both insulin resistance in target tissues and impaired insulin secretion from pancreatic beta cells; the location of the primary defect remains the source of much debate [1-3]. The defect in secretion may be specific for glucose and has been shown to affect the normal biphasic secretory pattern with loss of first phase release. In contrast, the response to non-nutrient secretagogues is retained [3]. Our current understanding is that in order for glucose 
to stimulate insulin release it must first enter the pancreatic beta cells (by GLUT 2 transporters) and be metabolised via the glycolytic and mitochondrial oxidative pathways. The resulting ATP generated is thought to close ATP-sensitive $\mathrm{K}+$ channels which in turn triggers membrane depolarisation, $\mathrm{Ca}^{2+}$ entry and hence exocytosis [4-6]. It is not clear however, how increased flux through the metabolic pathway and the ensuing changes are related to the biphasic secretory characteristic produced by glucose stimulation. In the present study, we have examined the GK (Goto-Kakizaki) Wistar rat, a non-obese, spontaneous model of NIDDM which was derived from normal Wistar rats by using impaired glucose tolerance as a selection index $[7,8]$. These animals have been shown to exhibit impaired insulin secretion in vivo or in vitro using the perfused pancreas technique and isolated pancreatic islets [7-10]. We have characterised insulin secretion from isolated pancreatic islets in response to nutrient and non-nutrient secretagogues. To investigate the role of impaired islet function in the development of diabetes in this model, we have examined the secretory responses from islets isolated from animals at different ages. Our approach was to first characterise insulin secretion in islets from adult-diabetic GK Wistar rats and then study islet function in islets isolated from progressively younger animals. The surprising finding in the first group studied at 8 weeks of age was that the secretory response to glucose was normal.

\section{Materials and methods}

Materials. Bovine serum albumin (BSA), hexokinase, glucose 6-phosphate dehydrogenase, ATP and NADP were supplied by Boehringer Mannheim Ltd. (Lewes, East Sussex, UK). Collagenase type 1A, glycerol, Hanks' balanced salts solution, U$\left[{ }^{14} \mathrm{C}\right]$ glucose $(370 \mathrm{mCi} / \mathrm{mmol})$, D-glyceraldehyde, $\mathrm{KIC}$ and $\mathrm{me}-$ thyl benzethonium hydroxide were all supplied by Sigma Chemical Co., (Poole, Dorset, UK). $\left.{ }^{125} \mathrm{I}\right]$ insulin $(100 \mu \mathrm{Ci} / \mu \mathrm{g}), 2$ $\left[{ }^{3} \mathrm{H}\right]$ glycerol $(1.0 \mathrm{Ci} / \mathrm{mmol})$ and $5-\left[{ }^{3} \mathrm{H}\right]$ glucose $(15.7 \mathrm{Ci} / \mathrm{mmol})$ were supplied by Amersham International plc, (Aylesbury, Bucks., UK). Millicell culture inserts were supplied by Millipore, (Milton Keynes, Bucks., UK). Coomassie Blue dye-reagent for protein determination was supplied by Biorad Laboratories Ltd., (Watford, Herts, UK). Sagatal was supplied by Southern Veterinary Supplies, (Lewes, East Sussex, UK). Antirat insulin antibody was generously provided by Dr. R. Turner, (University of Oxford, Oxford, UK).

Animals. A colony of GK Wistar rats was established at the animal facility, St. Mary's Hospital Medical School in September 1992 from two male and seven female animals supplied by Dr. K.-I. Suzuki, (Tohoku Medical School, Sendai, Japan). Animals were selected at either 8 or 14 weeks of age for the experiments outlined below. Controls were age-matched Wistar rats supplied by Harlan Olac Bicester, Oxon, UK. All animals were allowed free access to food and drink.

Glucose tolerance test. An intraperitoneal glucose tolerance test was performed on rats that had been fasted overnight using a dose of $2 \mathrm{~g}$ glucose per $\mathrm{kg}$ body weight. Rats were anaesthetised with $0.4 \mathrm{ml}$ sagatal and blood samples $(50 \mu \mathrm{l})$ collected from cut tail tips at intervals up to $2 \mathrm{~h}$. These were mixed with ice cold perchloric acid at a final concentration of $3.84 \%(\mathrm{v} / \mathrm{v})$, centrifuged for $5 \mathrm{~min}$ at $2500 \mathrm{~g}$ and supernatant samples collected and stored at $-20^{\circ} \mathrm{C}$ prior to assay using the hexokinase method on a Cobas Bio auto-analyser (Roche, Welwyn Garden City, UK). At the end of the $2-h$ period the rats were killed by cervical dislocation.

Islet isolation. Islets of Langerhans were prepared by collagenase digestion following injection of collagenase solution into the pancreas through the bile duct [11]. Rats were first anaesthetised with $0.4 \mathrm{ml}$ sagatal (intraperitoneal) and killed by exsanguination which facilitated collection of blood samples for glucose determination. Free islets were collected under a dissecting microscope with a $20-\mu l$ pipette into Hepes-buffered Hanks' medium containing $0.5 \%$ bovine serum albumin. The protein content of the islets was determined using the Biorad method [12].

Insulin release. Insulin release from freshly isolated islets was measured in a perifusion system as described elsewhere [13]. Islets (50) were housed in small chambers on Millicell culture inserts and perifused in basal medium (Krebs Ringer containing $20 \mathrm{mmol} / 1 \mathrm{Hepes}, \mathrm{pH} 7.4,5 \mathrm{mg} / \mathrm{ml} \mathrm{BSA}$ and $2 \mathrm{mmol} / 1$ glucose) for $60 \mathrm{~min}$ at a flow rate of $1 \mathrm{ml} / \mathrm{min}$ at $37^{\circ} \mathrm{C}$ prior to collection of fractions. Test agents were added as indicated. Fractions $(2 \mathrm{ml})$ were collected at 2 -min intervals and stored at $-20^{\circ} \mathrm{C}$ prior to assay for insulin by RIA [14]. At the end of the perifusion islet insulin was released by sonication and samples collected and stored for determination of total insulin content.

Insulin release in static incubations was measured from five islets in tubes containing $0.6 \mathrm{ml}$ of Hepes-buffered Krebs Ringer buffer containing $2 \mathrm{mg} / \mathrm{ml}$ BSA and various glucose concentrations. Incubations were stopped after $1 \mathrm{~h}$ at $37^{\circ} \mathrm{C}$ by cooling on ice and after centrifugation, aliquots were collected for insulin assay.

Glucose metabolism in isolated islets. The rate of glucose metabolism was measured in 10 islets in Hepes-buffered Krebs Ringer buffer containing $2 \mathrm{mg} / \mathrm{ml} \mathrm{BSA}$ at various glucose concentrations. Glucose utilisation and glucose oxidation were measured using $5-\left[{ }^{3} \mathrm{H}\right]$ glucose and $\mathrm{U}-\left[{ }^{14} \mathrm{C}\right]$ glucose, respectively as previously described [15].

$2-\left[{ }^{3} \mathrm{H}\right]$ Glycerol metabolism in isolated islets. The rate of $2-\left[{ }^{3} \mathrm{H}\right]$ glycerol metabolism (final concentration, $200 \mu \mathrm{mol} / 1$ ) to $\left[{ }^{3} \mathrm{H}\right]$ $\mathrm{H}_{2} \mathrm{O}$ was measured in 10 islets in Hepes-buffered Krebs Ringer buffer containing $2 \mathrm{mg} / \mathrm{ml}$ BSA at 2 and $16 \mathrm{mmol} / 1$ glucose concentrations as described by Giroix et al. [16]. $\left[{ }^{3} \mathrm{H}\right] \mathrm{H}_{2} \mathrm{O}$ was collected as described previously [15].

\section{Statistical analysis}

Data are presented as the mean \pm SEM for the number of experiments indicated. Statistical analysis was carried out using Student's $t$-test.

\section{Results}

Animals. The characteristics of the rats used in this study are summarised in Table 1 . The body weights of GK Wistar rats were similar to controls at 8 weeks of 
Table 1. Characteristics of control and GK Wistar rats

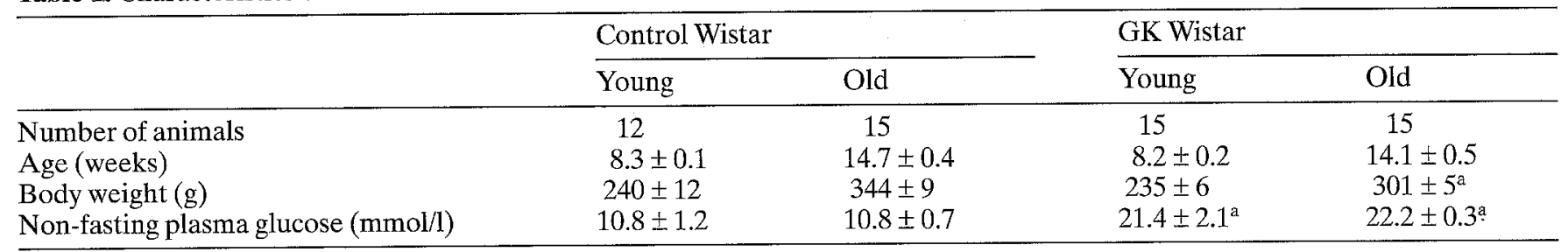

${ }^{\mathrm{a}} p<0.001$ compared to controls

Table 2. Insulin and protein content of isolated islets

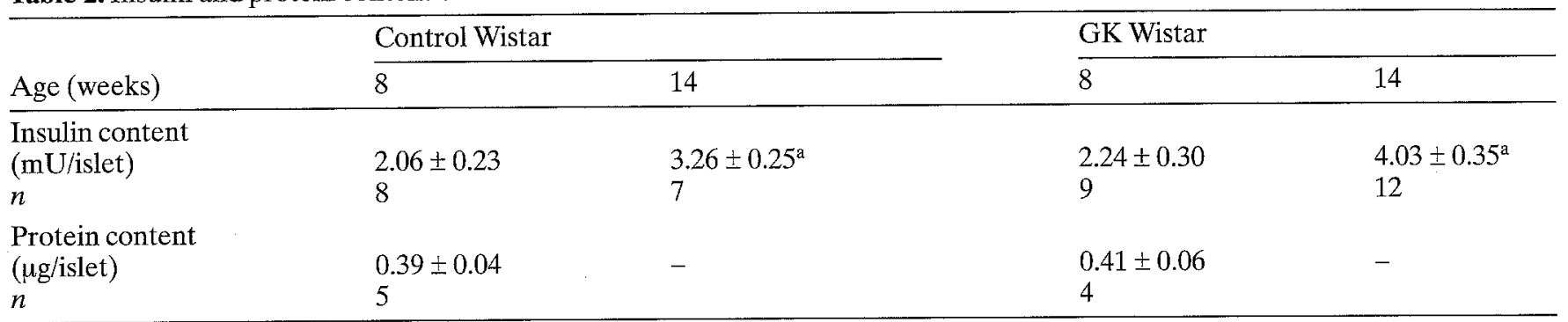

${ }^{a} p<0.01$ compared to insulin content of islets from young rats

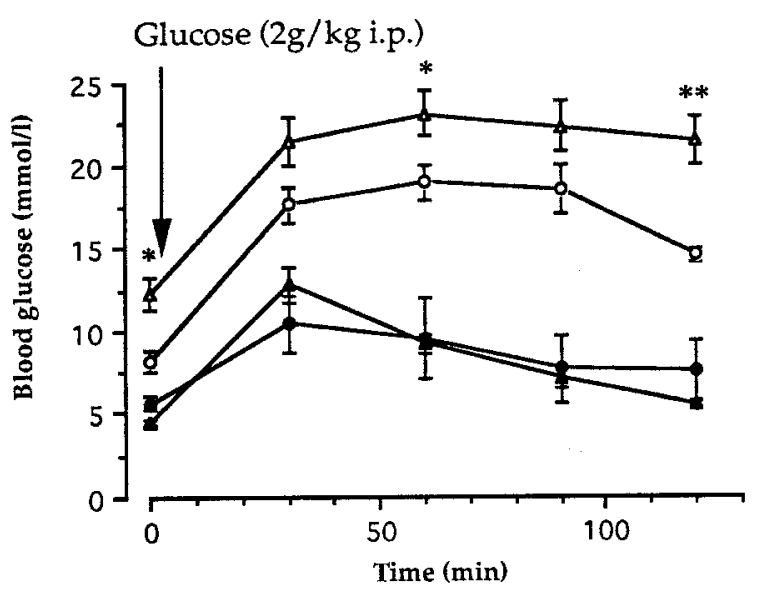

Fig. 1. Glucose tolerance in response to administration of glucose $(2 \mathrm{~g} / \mathrm{kg}$ body weight, i.p.) in either control $(\boldsymbol{\bullet}, \boldsymbol{\Delta})$ or GK Wistar rats $(0, \triangle)$ at $8(\bullet, 0)$ or 14 weeks of age $(\boldsymbol{\Delta}, \triangle)$ Wistar rats. Data represent the mean \pm SEM of data from 4-5 animals. $* p<0.05, * * p<0.01$ compared to 8-week-old GK Wistar rats (Student's $t$-test)

age but were significantly reduced by 14 weeks of age. Fasting and non-fasting plasma glucose levels of GK Wistar rats were significantly higher than in controls at either age (Table 1 and Fig. 1). The high plasma glucose levels in both control and diabetic animals reflect the fact that the animals were maintained under anaesthetic or had undergone surgery as part of the islet isolation procedure prior to collection of samples. After intraperitoneal administration of glucose solution ( $2 \mathrm{~g} / \mathrm{kg}$ body weight), blood glucose in GK Wistar rats increased to significantly higher levels than in control animals and did not return to basal values. Blood glucose levels of 14-week-old GK Wistar rats were significantly higher than those of 8 -week-old GK Wistar rats during the glucose tolerance test.

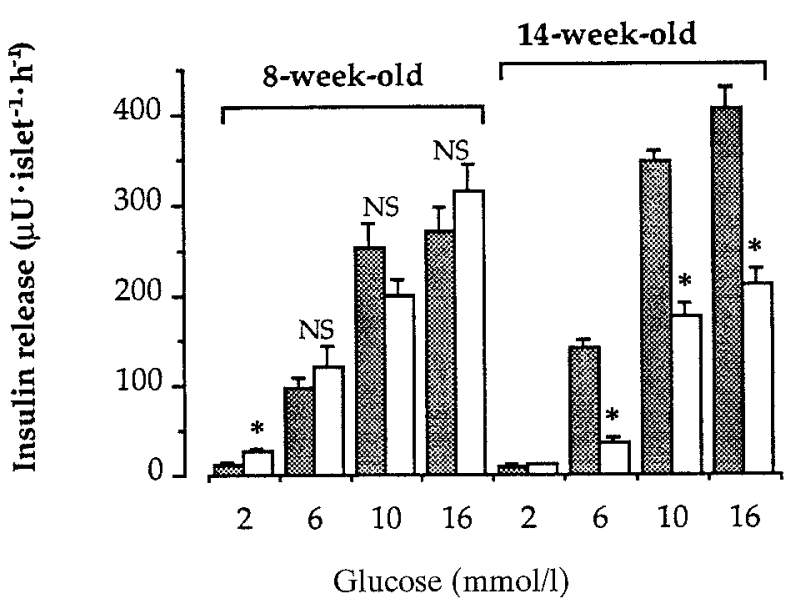

Fig. 2. Glucose induced-insulin release in islets from control and GK Wistar rats aged 8 and 14 weeks. Islets (5) from control (ם) or GK Wistar rats ( $\square$ ) were incubated with various concentrations of glucose for $1 \mathrm{~h}$ at $37^{\circ} \mathrm{C}$. Results are presented as $\mu U$ of insulin released per islet per $h$ and represent the mean \pm SEM of 16-18 observations from four separate experiments. ${ }^{*} p<0.001$ compared to control insulin release at the same glucose concentration; NS, not significant (Student's $t$ test)

Insulin release. Islets collected from pancreatic digests of GK Wistar rats were similar in size to those from controls on visual inspection and this was confirmed by determination of the protein and insulin content both of which were similar in GK Wistar and control islets (Table 2). Insulin content significantly increased with age in both control and GK Wistar rat-islets $(p<0.01)$. Insulin release was assessed in static incubations in response to varying medium glucose concentrations (Fig.2). Raising the medium glucose concentration increased insulin release above basal secretion in a dose-dependent manner. Maximal re- 
lease was 25 and 40 fold greater than basal in islets isolated from 8 and 14-week-old control rats, respectively. In islets isolated from 14-week-old GK Wistar rats, glucose stimulated insulin release was significantly impaired at all stimulatory concentrations tested. The insulin released ranged between $25-52 \%$ of that secreted by control islets. In contrast, in islets from 8-week-old GK Wistar rats, insulin secretion was not significantly different to that of controls when the glucose concentration in the incubation medium was either 6,10 or $16 \mathrm{mmol} / \mathrm{l}$. However, at the basal glucose concentration ( $2 \mathrm{mmol} / \mathrm{l})$, insulin secretion was significantly two times higher than in control islets.

The responses of perifused islets from control and GK Wistar rats to stimulation by nutrient secretagogues are shown in Figure 3. Stimulation with glucose $(10 \mathrm{mmol} / \mathrm{l})$ elicited a biphasic secretory response in islets from either 8 or 14-week-old control rats; first phase release peaking 6-8 $\mathrm{min}$ after exposure to the hexose at 20 times greater than basal secretion. The rising second phase of release was sustained for the duration of exposure to glucose and total insulin released during this period was 10 times greater than that released during the first phase. Data from islets of 8 -week-old controls only are presented. In islets isolated from 14-week-old GK Wistar rats, the secretory response to glucose was significantly impaired with loss of both first phase $(0.4 \pm 0.1$ in GK compared with $4.3 \pm 1.3 \mu \mathrm{U}$ per islet in controls during $0-10 \mathrm{~min}$ stimulatory period, $p<0.01$ ) and second phase release $(13.0 \pm 1.2$ in GK compared with $54.4 \pm 15.1 \mu \mathrm{U}$ per islet in controls during the 11-35 min stimulatory period, $p<0.05)$. The response to a second stimulation with arginine $(19 \mathrm{mmol} / \mathrm{l})$ was similar to that of islets from 14-week-old control animals. In contrast, the secretory response to stimulation with glucose in islets from 8-week-old GK Wistar rats was not significantly different to that seen in control islets. These were $2.1 \pm 0.6$ in GK compared with $3.3 \pm 1.4 \mu \mathrm{U}$ per islet in controls during the $0-10 \mathrm{~min}$ stimulatory period and $26.4 \pm 6.0$ in GK compared with $39.9 \pm 7.4 \mu \mathrm{U}$ per islet in controls during the 11-35 min stimulatory period. The secretory responses to perifusion with medium containing D-glyceraldehyde $(10 \mathrm{mmol} / \mathrm{l})$ or $\mathrm{KIC}$ $(10 \mathrm{mmol} / \mathrm{l})$ are also shown in Figure 3, panel B. In islets isolated from 14-week-old GK Wistar rats, KIC elicited a biphasic secretory response similar to that observed in controls. In contrast, the secretory response to stimulation by glyceraldehyde was virtually absent in islets isolated from either 14-week-old (data not shown) or 8-week-old diabetic animals (Fig. 3).

Glucose metabolism. The effect of changing the medium glucose concentration on the rate of glucose utilisation and oxidation in islets is shown in Figure 4. Raising the medium glucose concentration to $16 \mathrm{mmol} / \mathrm{l}$ significantly increased rates of glucose utilisation and oxidation in islets isolated from 8-week-

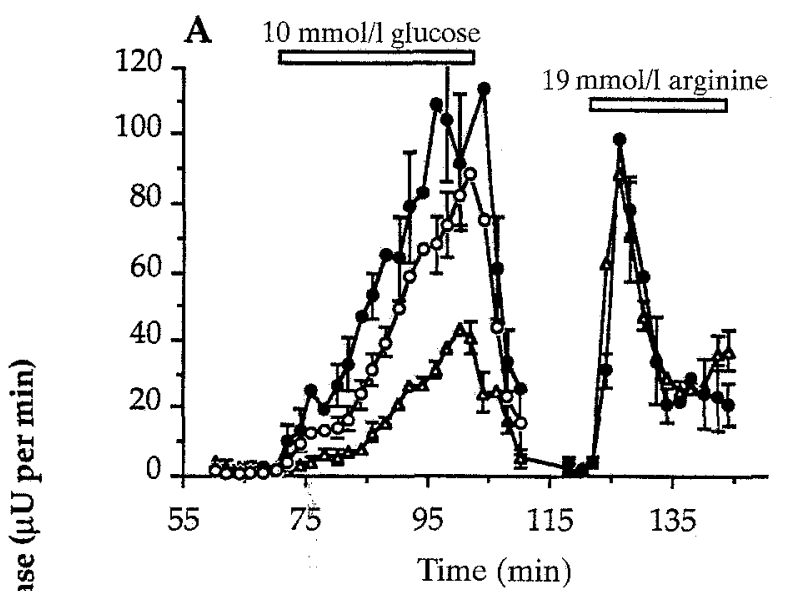

B

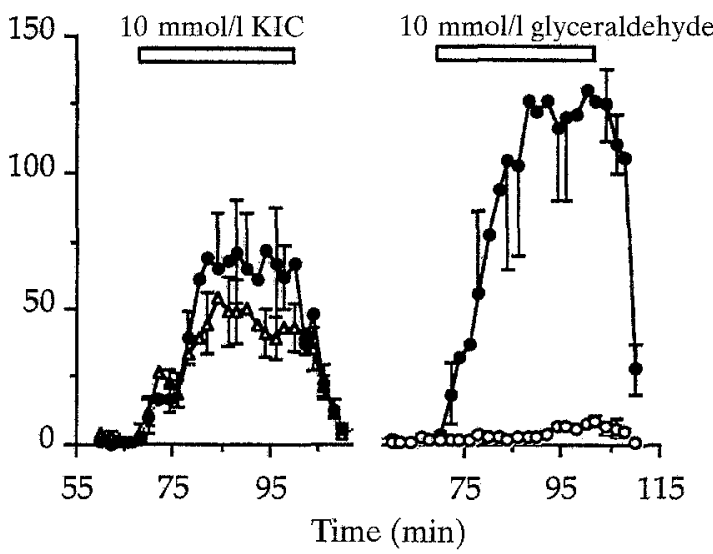

Fig.3. (A, B) Insulin release in response to nutrient secretagogues in perifused islets from control and GK Wistar rats aged either 8 or 14 weeks. 50 islets from either $8(0)$ or 14-week-old $(\triangle)$ GK Wistar rats or 8-week-old controls $(\bullet)$ were perifused with basal medium at $2 \mathrm{mmol} / \mathrm{l}$ glucose for $65 \mathrm{~min}$ prior to stimulation with $10 \mathrm{mmol} / \mathrm{l}$ glucose followed by $19 \mathrm{mmol} / \mathrm{l}$ arginine (panel A). The responses to stimulation by $10 \mathrm{mmol} / \mathrm{gly}$ ceraldehyde in islets from 8 -week-old control ( ) or GK Wistar rats $(O)$ and to stimulation by $10 \mathrm{mmol} / 1 \mathrm{KIC}$ in islets from 14 week-old control $(\bullet)$ or GK Wistar rats $(\triangle)$ are shown in panel B. Data are expressed $\mu \mathrm{U}$ insulin released per min and represent the mean \pm SEM for 3-6 separate experiments

old control and GK Wistar rats, respectively. In islets from GK Wistar rats, the rates of glucose utilisation were significantly higher at all glucose concentrations tested whereas the rates of glucose oxidation were not significantly different with the exception of incubations at basal glucose $(2 \mathrm{mmol} / \mathrm{l})$ where the rate was significantly $(50 \%)$ higher than in control islets. When the data were expressed as percentage paired ratio (rate of oxidation/rate of utilisation), the values were significantly lower in islets from GK Wistar rats at all glucose concentrations tested. Raising the medium glucose concentration above $2 \mathrm{mmol} / 1$ significantly increased the paired ratio in islets from controls at all stimulatory glucose concentrations whereas this effect was only observed at $6 \mathrm{mmol} / \mathrm{l}$ glucose in islets from GK Wistar rats. 


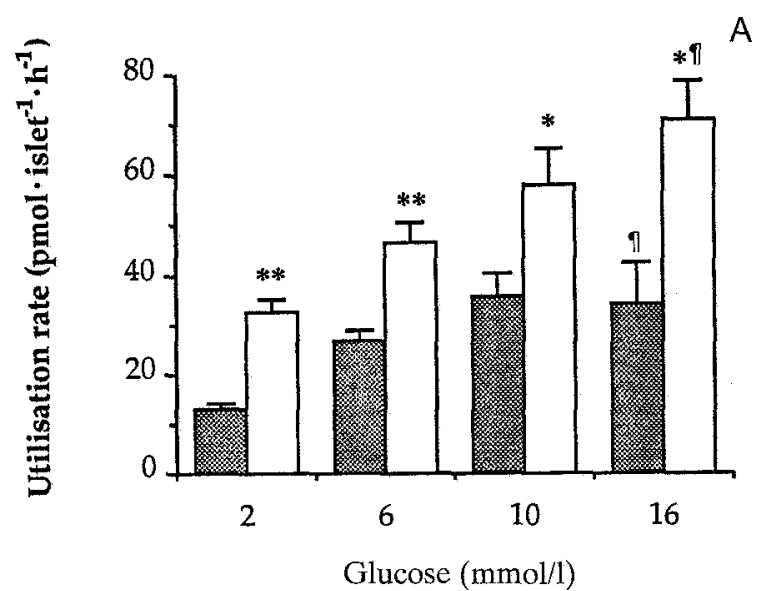


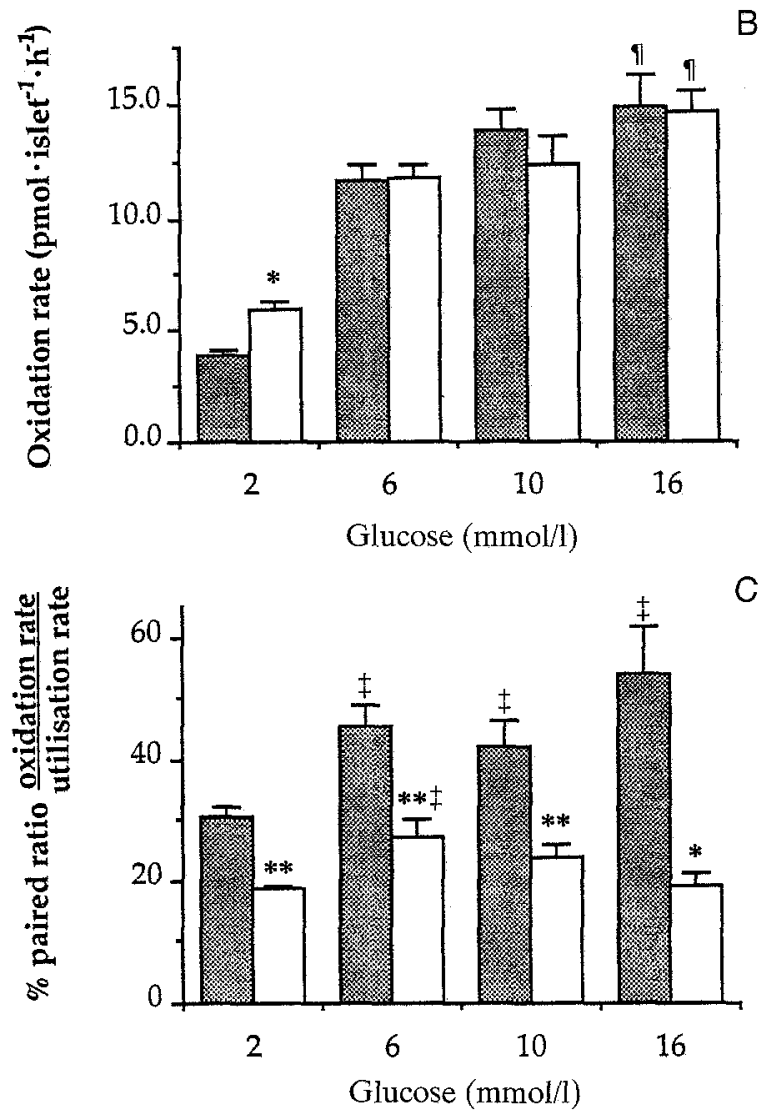

Fig. 4 (A-C) Glucose metabolism in islets from 8-week-old control and GK Wistar rats. Groups of 10 islets isolated from control ( $)$ or GK Wistar rats $(\square)$ were incubated for $2 \mathrm{~h}$ at $37^{\circ} \mathrm{C}$ with various concentrations of glucose and $5-\left[{ }^{3} \mathrm{H}\right]$ glucose and $U-\left[{ }^{14} \mathrm{C}\right]$ glucose to measure glucose utilisation (panel $\mathrm{A}$ ) or oxidation (panel B), respectively. Data are expressed as pmoles produced per islet per $h$ and represent the mean \pm SEM of $10-$ 12 observations from three separate experiments. In panel $\mathrm{C}$ data are presented as the percentage paired ratio (rate of oxidation/rate of utilisation). $* p<0.05, * * p<0.001$ compared to controls; $+p<0.05, \quad p<0.01$ compared to $2 \mathrm{mmol} / 1$ glucose (Student's $t$-test)

$2-\left[{ }^{3} \mathrm{H}\right]$ Glycerol metabolism. The effect of increasing the medium glucose concentration from 2 to $16 \mathrm{mmol} / 1$ on the rate of $2-\left[{ }^{3} \mathrm{H}\right]$ glycerol conversion to ${ }^{3} \mathrm{H}_{2} \mathrm{O}$ in isolated islets from 8-week-old control and GK Wistar
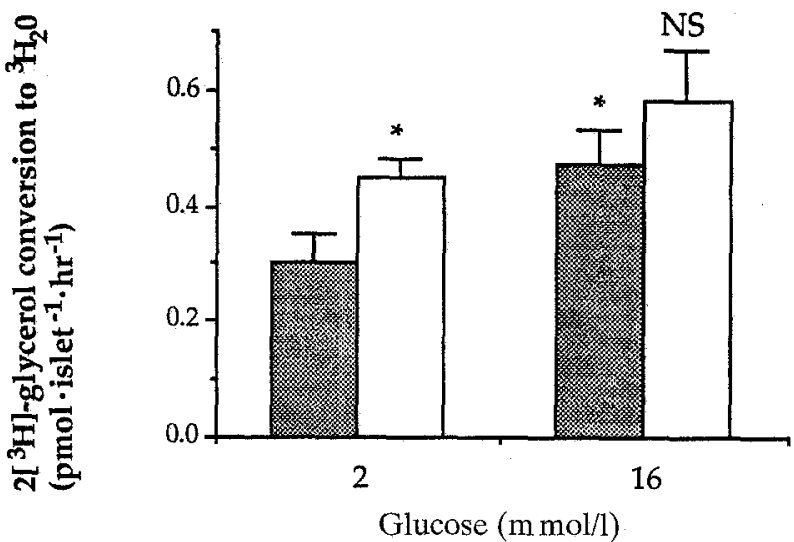

Fig. 5. Glycerol metabolism in islets from 8-week-old control and GK Wistar rats. Groups of 10 islets isolated from control (घ) or GK Wistar rats $(\square)$ were incubated for $2 \mathrm{~h}$ at $37^{\circ} \mathrm{C}$ with either 2 or $16 \mathrm{mmol} / 1$ glucose and $2-\left[{ }^{3} \mathrm{H}\right]$ glycerol $(0.2 \mathrm{mmol} / \mathrm{l})$. Data are expressed as pmoles $\left[{ }^{3} \mathrm{H}\right]-\mathrm{H}_{2} \mathrm{O}$ produced per islet per hour and represent the mean \pm SEM of eight observations from four separate experiments. $* p<0.05$, compared to controls at $2 \mathrm{mmol} / \mathrm{l}$ glucose; (Student's $t$-test)

rats is shown in Figure 5. 2- $\left[{ }^{3} \mathrm{H}\right]$ glycerol metabolism was increased by raising the medium glucose concentration in islets from both control and GK Wistar rats although this was only significant in the case of the controls. The rate of $2-\left[{ }^{3} \mathrm{H}\right]$ glycerol metabolism at $2 \mathrm{mmol} / \mathrm{l}$ glucose was significantly higher in islets from GK Wistar rats than in controls.

\section{Discussion}

It has previously been demonstrated in animal models of NIDDM that hyperglycaemia or some factor associated with the diabetic condition perturbs metabolic function in pancreatic islets $[1,17-19]$. In studies to identify defective processes responsible for the development of diabetes in these models it is therefore important to determine whether the expression of the defect precedes or is a consequence of the diabetic state. A major finding in the present study is that in islets isolated from young GK Wistar rats aged 8 weeks, an almost normal secretory response to glucose was observed in both perifusion and static incubations. This was found despite the fact that diabetes is already clearly established in these animals [7, $20]$ and is present from the age of 4 weeks at least [7,9]. These observations would suggest that an intrinsic islet defect in the glucose stimulus-secretory pathway is not a primary factor in the pathogenesis of the diabetes of GK Wistar rats.

Our finding of normal secretory function may seem incompatable with the presence of normal insulin content in islets of diabetic 8-week-old GK Wistar rats since hyperglycaemia should chronically stimulate and deplete insulin stores in the islets. These observations may be reconciled by the finding that islets from 
GK Wistar rats exhibit increased pro-insulin synthesis [21] which may maintain their insulin content. Additionally, it has been shown that glucose-stimulated insulin secretion in the perfused pancreas is more severely impaired than in isolated islets in models of diabetes including the GK Wistar rat [10, 22]. This in turn suggests that circulatory or intra-pancreatic factors contribute to the inhibition of insulin release [22] and that this effect is lost on isolation of islets. Thus, the function of islets in situ may be impaired in comparison with isolated islets. It follows from this that our findings do not necessarily conflict with the data presented by Ohneda and coworkers [20] who showed that insulin release in response to stimulation by glucose was impaired in perfused pancreas from 8-weekold GK Wistar rats. Additionally, these authors showed that islet volume per pancreas in 12-week-old GK Wistar rats was half that of controls. This in turn is consistent with our own observation that digestion of pancreata of GK Wistar rats of all ages yields a reduced number of islets compared to controls (S.J.Hughes, unpublished observations). Furthermore, the total pancreatic-insulin content has previously been shown to be reduced in 10-week-old GK Wistar rats [9]. Taken together these observations suggest that reduced pancreatic islet content may contribute to the reduced insulin output in perfused pancreata of 8-week-old GK Wistar rats [20] rather than impaired secretory function of individual islets. Thus, reduced insulin output may play an important contribution in the development of diabetes in these rats despite normal islet function.

Recent studies have documented the impaired secretory responses of islets isolated from GK Wistar rats in static incubations $[10,21]$ whereas here we also report experiments using perifused islets. Our experiments with islets isolated from adult 14-week-old GK Wistar rats in static incubations confirm that glucose in unable to elicit a normal secretory response in older animals [21]. In one study [10] however, secretory responses were impaired in islets isolated from GK Wistar rats of unspecified age but weighing 200-250 g which coincides with the weights of 8 -week-old animals used in this study. The reason for the apparent discrepancy in the secretory data is unclear; the maximal secretory responses to stimulation with $16 \mathrm{mmol} / \mathrm{l}$ glucose were 2 and 5 fold greater than basal in islets from diabetic and control animals, respectively, in this study [10]. In the present study, the maximal response was 19 times greater than basal in islets from 14-weekold animals although this was considerably poorer than the 45 fold stimulation of secretion observed in control islets. No study to date however, including this one has measured insulin release in islets challenged with the maximally stimulating glucose concentration $(30 \mathrm{mmol} / \mathrm{l})$ in order to assess the maximum velocity of the secretory rate. The perifusion studies show that the defect in glucose-stimulated insulin release re- duces both first and second phases of secretion although the first phase appeared more severely affected. This impairment is clearly not related to reduced insulin content of islets since in the present study insulin content was similar in control and GK Wistar rat islets at variance with previous findings $[10$, 21]. This discrepancy probably arises because in these studies smaller islets were isolated from GK Wistar rats than from controls as indicated by DNA content $[10,21]$. This in turn may result from different islet isolation protocols in comparison with the one used here. In addition, islets from 14-week-old GK Wistar rats were able to respond normally to stimulation by the non-nutrient secretagogue arginine and the nutrient secretagogue KIC. This latter finding supports the conclusion of Giroix et al. [21] that islets from GK Wistar rats do not exhibit a generalised impairment in mitochondrial function although these authors have proposed that impaired electron transport chain function may prevail in the islet mitochondria.

Islets isolated from 17-week-old GK Wistar rats exhibit impaired mitochondrial oxidation of glucose $[21,23]$. In islets isolated from 8-week-old control and GK Wistar rats no difference in the rate of glucose oxidation was apparent across the range of stimulatory glucose concentrations tested. In the present study $\mathrm{U}-\left[{ }^{14} \mathrm{C}\right]$ glucose was used to assess glucose oxidation as used in one other study [10] rather than 6$\left[{ }^{14} \mathrm{C}\right]$ glucose $[21,23]$. Using $6-\left[{ }^{14} \mathrm{C}\right]$ glucose allows assessment of the oxidation in the Krebs cycle of glucose-derived acetyl residues. Using $U_{-}\left[{ }^{14} \mathrm{C}\right]$ glucose also allows measurement of the decarboxylation of pyruvate and thus reflects the total oxidative capacity of glucose. Surprisingly, the rates of glucose utilisation at all incubation conditions were significantly higher in islets from diabetic animals. This agrees with one of the studies above [10] in which elevated glucose utilisation was attributed to increased anaerobic glucose metabolism. When our data were expressed as a paired ratio (rate of oxidation/rate of utilisation), the values for GK Wistar rat islets were significantly reduced - an observation which reflects the high rates of utilisation. It could be argued that islets from these animals fail to metabolise via mitochondrial oxidation the normal percentage of glycolytic products. This might suggest an impairment in mitochondrial function. It should be emphasised however that (theoretically) islets from GK Wistar rats should generate sufficient ATP (a key metabolic coupling factor) to sustain normal glucose-stimulated insulin secretion. Indeed, it might be expected that the higher than normal rates of glycolysis (as indicated by the higher rates of glucose utilisation) in these islets would sustain higher rates of secretion than in controls. The rather poorer rates of glucose-stimulated insulin secretion actually observed in perifusion experiments might indicate that coupling of glucose metabolism to membrane depolarisation and insulin secretion is not fully 
intact in islets from 8-week-old GK Wistar rats. This may reflect the increased glucose cycling previously reported [10]. Alternatively, it has been suggested that the impaired mitochondrial oxidation of glucose results at least in part from reduced activity of FADlinked glycerophosphate dehydrogenase [24]. The finding in the present study that glyceraldehyde fails to promote any secretion of insulin in islets from these animals might reflect an impairment in this pathway. Measurement of 2-[3 $\left.{ }^{3} \mathrm{H}\right]$ glycerol metabolism in islets from 8-week-old GK Wistar rats indicated that glycerol phosphate shuttle activity was not impaired in islets from the diabetic animals. In fact, $2-\left[{ }^{3} \mathrm{H}\right]$ glycerol metabolism was significantly higher in incubations under basal conditions paralleling the increased rate of glucose utilisation in the islets. The impaired response to glyceraldehyde may alternatively result from a defect in glycolysis distal to glyceraldehyde 3phosphate although given the either high or normal rates of glucose utilisation reported here and elsewhere $[10,21,23]$, this seems unlikely. It is more plausible that the failure of glyceraldehyde to stimulate insulin release in these islets results from a failure of the triose to enter the glycolytic pathway and hence metabolism because of an impairment in the function of the enzyme-triose kinase.

A defect in FAD-linked glycerophosphate dehydrogenase has been identified in islets of other models of diabetes such as rats injected with streptozotocin in the neonatal period [25] and db/db mice [26]. As this abnormality appears in several models of diabetes which result from either distinctive genetic backgrounds or chemical treatment, it is possible that the defect is the result of some factor associated with diabetes rather than a primary cause. The measurements made in the present study of glycerol phosphate shuttle activity in islets from 8-week-old GK Wistar rats support this conclusion. Similarly, reduced expression of GLUT 2 transporters has also been found in islets from several models of diabetes including rats injected with streptozotocin as neonates, male Zucker diabetic (ZDF/Drt-fa[F10]) rats and GK Wistar rats [20, 27, $28]$. In the latter case, it was concluded that the reduction in expression of transporters was of insufficient magnitude to perturb glucose metabolism and insulin secretion in the islets [20]. In the present study, the deterioration in the secretory response to glucose with age in GK Wistar rats suggests that abnormalities in islet function develop as a consequence of the prevailing diabetic state which in turn leads to impaired insulin release. This would then contribute to the increasing hyperglycaemia as indicated by the poorer glucose tolerance observed in older rats seen here and previously [9].

In summary, we have characterised the insulin secretory responses of islets isolated from control and GK Wistar rats aged 8 and 14 weeks. The data show that in islets of older animals the secretory response to glucose is impaired with loss of first and second phase release whereas the responses to other non-nutrient (arginine) and nutrient (KIC) secretagogues remain intact. In islets from 8-week-old GK Wistar rats, the secretory response to glucose was marginally poorer but not significantly different to that seen in controls despite the fact that these animals were diabetic. Thus, the reduced insulin output from perfused pancreata of 8-week-old GK Wistar rats [20] probably results from reduced pancreatic islet content rather than impaired secretion of individual islets or the inhibitory effects of extrapancreatic factors which are lost on islet isolation $[10,22]$. Abnormalities in function were apparent in islets from 8-week-old animals. These were an impaired secretory response to glyceraldehyde and high rates of glucose utilisation concomitant with unimpaired glucose oxidation and glycerol phosphate shuttle activity. The enhanced rate of glucose utilisation probably reflects increased anaerobic metabolism [10] whereas the defective glyceraldehyde response probably reflects impaired entry of the triose into glycolysis. Our data would suggest that a defect in the glucose signalling pathway in beta cells is not a primary cause of the diabetes of GK Wistar rats and that deterioration of the secretory response is the consequence of some factor associated with the diabetic state.

Acknowledgements. The assistance of M.Wilson and R. Nithyanathan is gratefully appreciated. This study was supported by a grant from the British Diabetic Association.

\section{References}

1. Leahy JL (1990) Natural history of beta cell disfunction in NIDDM. Diabetes Care 13: 992-1010

2. DeFronzo RA (1992) Pathogenesis of type 2 diabetes mellitus: a balanced overview. Diabetologia 35: 389-397

3. Porte D (1991) Beta cells in type 2 diabetes mellitus. Diabetes 40: 166-180

4. Prentki M, Matschinsky FM (1987) Ca2+, cyclic AMP and phospholipid derived messengers in coupling mechanisms of insulin secretion. Physiol Rev 67: 1185-1248

5. Arkhammar P, Nilsson T, Rorsman P, Berggren P-O (1987) Inhibition of ATP-regulated $\mathrm{K}+$ channels precedes depolarisation-induced increase in cytoplasmic free calcium concentration in pancreatic beta cells. J Biol Chem 262: $5448-5454$

6. Ashcroft FM, Ashcroft SJH (1990) Properties and functions of ATP-sensitive K+ channels. Cell Signal 2: 197-214

7. Goto Y, Suzuki KI, Sasaki M, Ono T, Abe S (1988) GK rat as a model of nonobese noninsulin-dependent diabetes: selective breeding over 35 generations. In: Shafrir E, Renold AE (eds) Frontiers of diabetes research. Lessons from animal diabetes II. Libbey press, London pp 301-303

8. Kimura K, Toyota T, Kakizaki M, Kudo M, Takebe K, Goto $Y(1982)$ Impaired insulin secretion in the spontaneous diabetes rats. Tohoku J Exp Med 137: 453-459

9. Portha B, Serradas P, Bailbe D, Suzuki K-I, Goto Y, Giroix M-H (1991) Beta cell insensitivity to glucose in the GK rat, a spontaneous nonobese model of type 2 diabetes. Diabetes 40: 486-491 
10. Ostenson C-G, Khan A, Abdel-Halim SM et al. (1993) Abnormal insulin secretion and glucose metabolism in pancreatic islets from the spontaneously diabetic GK rat. Diabetologia $36: 3-8$

11. Sutton R, Peters M, McShane P, Gray DWR, Morris PJ (1986) Isolation of rat pancreatic islets by ductal injection of collagenase. Transplantation 42: 689-691

12. Bradford MM (1976) A rapid and sensitive method for the quantitation of microgram quantities of protein utilizing the principle of protein-dye binding. Anal Biochem 72: 248-254

13. Hughes SJ, Carpinelli A, Niki I, Nicks J, Ashcroft SJH (1992) Stimulation of insulin release by vasopressin in the clonal B-cell line HIT-T15: the role of protein kinase C. J Mol Endocrinol 8: 145-153

14. Ashcroft SJH, Crossley JR (1974) Effects of glucose, nacetylglucosamine, glyceraldehyde and other sugars on insulin release in vivo. Diabetologia 11:279-284

15. Hughes SJ (1994) The role of reduced glucose transporter content and glucose metabolism in the immature secretory responses of fetal rat pancreatic islets. Diabetologia 37:134140

16. Giroix M-H, Rasschaert J, Sener A et al. (1992) Study of hexose transport, glycerol phosphate shuttle and Krebs cycle in islets of adult rats injected with streptozotocin during the neonatal period. Mol Cell Endocrinol 83: 95-104

17. Leahy JL, Cooper HE, Deal DA, Weir GC (1986) Chronic hyperglycemia is associated with impaired glucose influence on insulin secretion: a study in normal rats using chronic in vivo glucose infusions. J Clin Invest 77: 908-915

18. Laury MC, Takao F, Bailbe D et al. (1991) Differential effects of prolonged hyperglycemia on in vivo and in vitro insulin secretion in rats. Endocrinology 128: 2526-2533

19. Thibault C, Laury MC, Bailbe D, Ktorza A, Portha B (1992) Effects of prolonged in vivo glucose infusion on insulin secretion in rats with previous glucose intolerance. Endocrinology 130: 2521-2527
20. Ohneda M, Johnson JH, Inman LR et al. (1993) GLUT 2 expression and function in beta cells of GK rats with NIDDM. Diabetes 42: 1065-1072

21. Giroix M-H, Vesco L, Portha B (1993) Functional and metabolic perturbations in isolated pancreatic islets from the GK rat, a genetic model of non insulin-dependent diabetes. Endocrinology 132: 815-822

22. Weir GC, Leahy JL, Bonner-Weir S (1986) Experimental reduction of betal cell mass: implications for the pathogenesis of diabetes. Diabetes Metab Rev 2: 125-161

23. Giroix M-H, Sener A, Portha B, Malaisse WJ (1993) Preferential alteration of oxidative relative to total glycolysis in pancreatic islets of two rat models of inherited or acquired type 2 diabetes mellitus. Diabetologia 36: 305-309

24. Ostenson C-G, Abdel-Halim SM, Rasschaert J et al. (1993) Deficient activity of FAD-linked glycerophosphate dehydrogenase in islets of GK rats. Diabetologia 36: 722-726

25. Giroix M-H, Rasschaert J, Bailbe D et al. (1991) Impairment of glycerol phosphate shuttle in islets from rats with diabetes induced by neonatal streptozocin. Diabetes 40 : 227-232

26. Sener A, Herberg L, Malaisse WJ (1993) FAD-linked glycerophosphate dehydrogenase in pancreatic islets of mice with hereditary diabetes. FEBS Lett 316: 224-227

27. Thorens B, Weir GC, Leahy JL, Lodish HF, Bonner-Weir S (1990) Reduced expression of the liver/beta cell glucose transporter isoform in glucose insensitive pancreatic beta cells of diabetic rats. Proc Nat Acad Sci USA 87: 6492-6506

28. Orci L, Ravazzola M, Baetens D et al. (1990) Evidence that down-regulation of B-cell glucose transporters in NDDM may be the cause of diabetic hyperglycemia. Proc Nat Acad Sci USA 87: 9953-9957 